\title{
BUENAS PRÁCTICAS PREVENTIVAS PARA ESTUDIANTES UNIVERSITARIOS DE CIENCIAS DE LA SALUD QUE REALICEN PRÁCTICAS ACADÉMICAS EXTERNAS
}

\author{
BEST PREVENTIVE PRACTICES FOR UNIVERSITY STUDENTS IN HEALTH SCIENCES CARRYING OUT \\ EXTERNAL ACADEMIC PRACTICES
}

\author{
Manuel Vaquero Abellán*, Pablo López Roldán, Fernando Vaquero González, José Checa Claudel, \\ Pilar Lora López, Silvia Portero de la Cruz, \\ Rosario Chacón Quintero \\ mvaquero@uco.es (*Corresponding Author)
}

Universidad de Córdoba

\begin{abstract}
External academic practices allow the application of theoretical knowledge, favoring the acquisition of competencies that prepare them for the exercise of professional activities, facilitate their employability and promote their entrepreneurship. Our objective has been to incorporate basic knowledge of occupational risk (OR) into the practical teaching of students of Health Sciences, to integrate risk prevention in the different professions, tasks and functions that they can exert after graduation. A computer application has been developed and implemented as an interactive tool for students to acquire basic competences in the area of OR, also a manual, which allows students to carry out their practices in a safe way.
\end{abstract}

Keywords: Students, Health Occupations; Best practices; Occupational Risks; Clinical Clerkship.

\section{Resumen}

Las prácticas académicas externas permiten aplicar conocimientos teóricos, favoreciendo la adquisición de competencias que les preparen para el ejercicio de actividades profesionales, faciliten su empleabilidad y fomenten su capacidad de emprendimiento. Nuestro objetivo ha sido incorporar a la docencia práctica de estudiantes de Ciencias de la Salud conocimientos básicos de prevención de riesgos (PRL), para integrar la prevención de riesgos en las diferentes profesiones, tareas y funciones que pueden ejercer después de graduarse. Se ha desarrollo e implementado una aplicación informática como herramienta interactiva para que el alumnado adquiera competencias básicas en materia de PRL, además de un manual, que permite a los estudiantes realizar sus prácticas de forma segura.

Palabras clave: Estudiantes del Área de la Salud; Buenas prácticas preventivas; Riesgos Laborales; prácticas clínicas.

\section{INTRODUCCIÓN}

Las universidades, como instituciones dedicadas a la enseñanza superior, tienen entre sus funciones esenciales, la preparación para el ejercicio de actividades profesionales que exijan la aplicación de conocimientos y métodos científicos y para la creación artística. Ello implica que la formación del estudiante universitario, no sólo debe realizarse sobre las competencias asociadas a los títulos oficiales, sino que deberán recibir enseñanzas en otras materias afines o transversales que permitan una preparación profesional con un enfoque -entre otros- de seguridad y salud laboral. La normativa, así lo pone de manifiesto a través del Estatuto del Estudiante Universitario, establece que los estudiantes tienen derecho a recibir formación sobre prevención de riesgos y a disponer de los medios que garanticen su salud y seguridad en el desarrollo de sus actividades de aprendizaje.

En este sentido, tanto la Estrategia Española de Seguridad y Salud en el Trabajo 2015-2020, como el Acuerdo del Consejo de Universidades de 7 de noviembre de 2011, por el que establecen directrices para la adaptación de la legislación de prevención de riesgos laborales a la universidad, ponen de manifiesto que la integración de la seguridad y salud en el trabajo en la educación universitaria es una necesidad prioritaria para consolidar una auténtica cultura preventiva. Es necesario concienciarse de que la prevención no comienza en el ámbito laboral, sino en etapas anteriores, en particular en el sistema educativo. Asimismo, requiere un apoyo y asistencia especial, a fin de conseguir profesionales cuya formación, además de garantizar la calidad de su trabajo, les permita realizarlo con las máximas garantías de seguridad.

Por todo ello, la Universidad de Córdoba (UCO) ha de promover la cultura preventiva mediante la potenciación de la integración transversal de la Prevención de Riesgos Laborales en los planes de estudio de todas las titulaciones universitarias. Actualmente esta integración es claramente insuficiente, muy variable y, en cualquier caso, no se aborda de forma sistemática con el objetivo de capacitar al alumnado para que desarrolle su actividad profesional considerando la seguridad y la salud de los trabajadores y aplicando los principios de la prevención de los riesgos laborales. Probablemente, muchas titulaciones universitarias que ya han adaptado sus planes de estudios Bolonia han desatendido la importancia que requiere la Prevención de Riesgos Laborales. 
Para paliar esta deficiente integración, la UCO, a través de su política preventiva, considera al alumnado como parte integrante de la Comunidad Universitaria, el cuál, debe ser destinatario de las políticas preventivas, informándose de los riesgos y de las medidas preventivas que les afecten e implicándose en el cumplimiento de las normas que se hayan dispuesto. Asimismo, empleando el Sistema de Gestión de la Prevención, la UCO debe facilitar que el alumnado adquiera los principios de seguridad y salud en el trabajo, estableciéndose las bases para su interiorización en todos los ámbitos de la vida.

Las prácticas académicas externas constituyen una actividad de naturaleza formativa realizada por los estudiantes universitarios y supervisada por las universidades, cuyo objetivo es permitir a los mismos aplicar y complementar los conocimientos adquiridos en su formación académica, favoreciendo la adquisición de competencias que les preparen para el ejercicio de actividades profesionales, faciliten su empleabilidad y fomenten su capacidad de emprendimiento.

Los objetivos que se pretenden alcanzar con la realización de las prácticas académicas externas son:

a) Contribuir a la formación integral de los estudiantes complementando su aprendizaje teórico y práctico.

b) Facilitar el conocimiento de la metodología de trabajo adecuada a la realidad profesional en que los estudiantes habrán de operar, contrastando y aplicando los conocimientos adquiridos.

c) Fomentar el desarrollo de competencias técnicas, metodológicas, personales y participativas.

d) Obtener una experiencia práctica que facilite la inserción en el mercado de trabajo y mejore su empleabilidad futura.

e) Favorecer los valores de la innovación, la creatividad y el emprendimiento.

Centrándonos en la rama de Ciencias de la Salud, se ha detectado una importante carencia formativa en el alumnado que realiza prácticas académicas externas o a través de las asignaturas de practicum. Dicha carencia formativa se basa en que los estudiantes no poseen los conocimientos básicos para desarrollar sus actividades prácticas en condiciones óptimas de seguridad y salud.

\section{OBJETIVOS}

Los objetivos planteados para la puesta en marcha de este proyecto educativo han sido:

- Dar a conocer al alumno el marco normativo vigente, conceptual y de responsabilidades en materia de Prevención de Riesgos Laborales.

- Explicar a los estudiantes la norma que desarrolla la integración de la PRL en las diferentes profesiones, tareas y funciones que pueden ejercer después de graduarse.

- Inculcar en el alumnado los distintos riesgos (seguridad, higiene, ergonomía y psicosociología) existentes en su lugar de prácticas, así como, las medidas preventivas que pueden aplicar a las tareas que realizan.

- Transmitir a los estudiantes un conjunto de buenas prácticas específicas en el ámbito de la prevención que pueden desempeñar cuando finalicen sus estudios universitarios.

- Incorporar a la docencia práctica conocimientos básicos de PRL, utilizando tecnologías de la información y la comunicación.

- Facilitar la incorporación del alumnado de la UCO al mercado laboral.

\section{Metodología}

La metodología ha consistido en el desarrollo e implementación de un Aplicativo Informático basado en las nuevas tecnologías como herramienta interactiva para que el alumnado adquiera los conocimientos básicos en materia de PRL. Además de un Manual básico que recoja, desde las especialidades de prevención de riesgos Seguridad en el Trabajo, Higiene Industrial, Ergonomía y Psicosociología Aplicada y Medicina/Enfermería del Trabajo -, los conocimientos mínimos para que los estudiantes realicen sus prácticas de forma segura.

La herramienta informática está basada en un entorno de gestión vía WEB y localizada, a través de un portal, en Internet. Recoge y mantiene información dinámica ofreciendo soluciones interactivas, a medida y bajo demanda, desde la Unidad Docente Salud Pública hacia los estudiantes en sus prácticas externas. Asimismo, es capaz de interactuar desde el punto de vista del administrador y desde el punto de vista del usuario final.

Se ha desarrollado un site a través de una programación propia y exclusiva para nuestros requerimientos, basado en el concepto de gestor de contenidos. Éste es capaz de ofrecer cualquier tipo de contenido al participante, que 
puede ser de diversa naturaleza, en nuestro caso audio, video, texto, y con un área determinada de conocimiento: la formación del estudiante durante sus prácticas externas en los grados y másteres de Ciencias de la Salud.

Esta metodología favorece que el estudiante vaya formándose de manera autónoma, planteándose interrogantes, participando y asumiendo responsabilidades; $y$, por tanto, desarrollando la capacidad para aprender por sí mismo.

Para la evaluación, se cumplimentará un breve cuestionario para valorar la herramienta y los conocimientos adquiridos en esta materia por el alumnado.

Además, se realizarán sesiones programadas para poner en práctica los conocimientos adquiridos a través del entorno web.

\section{DESCRIPCIÓN}

Para alcanzar los objetivos de este Proyecto de Innovación Educativa se han realizado una serie de actividades planificadas durante el curso académico 2015/2016 en las siguientes fases:

Fase 1: Manual básico de Prevención de Riesgos y estudios complementarios.

- Revisión de fuentes bibliográficas que recojan posibles riesgos, así como buenas prácticas de control y prevención entre los estudiantes.

- Estudio sobre riesgos laborales a los que se encuentran expuestos los estudiantes.

- Elaboración de un manual básico de Prevención de Riesgos de Laborales y estudios complementarios para su difusión en formato papel.

- Guía de buenas prácticas preventivas y control de riesgos durante las prácticas académicas externas.

Fase 2: Elaboración de los vídeos didácticos y material interactivo.

- Elaboración de guion del "Estudio sobre riesgos laborales a los que se encuentran expuestos los estudiantes".

- Elaboración de guion de la "Guía de buenas prácticas preventivas y control de riesgos durante las prácticas académicas externas".

- Sesiones de grabación en videocámara.

Fase 3: Elaboración de la aplicación informática.

- La herramienta informática está basada en un entorno de gestión vía WEB y localizada, a través de un portal, en Internet.

- Simultáneamente se elabora un CD con la documentación del portal.

Para llevar a buen término todas las tareas, se ha contado con un equipo de trabajo multidisciplinar con amplia experiencia y conocimientos en materia de Prevención de Riesgos Laborales. Este equipo ha estado formado por médico del trabajo, ingeniero, enfermeras con el máster de PRL y técnicos de PRL e Informática con amplia experiencia laboral.

\section{RESULTADOS OBTENIDOS}

Con la ejecución de este proyecto, se han obtenido los siguientes resultados:

1) Un manual básico de PRL para estudiantes de grados y másteres de las ramas de Ciencias de la Salud.

2) Una aplicación informática que sirve como herramienta para que el alumnado adquiera los conocimientos básicos en materia de PRL.

Asimismo, con la implementación de ambos recursos, los egresados de estas titulaciones de la UCO habrán adquirido los conocimientos básicos en materia de seguridad y salud laboral para facilitar su incorporación al mercado de trabajo. 


\subsection{MANUAL BÁSICO DE PREVENCIÓN DE RIESGOS LABORALES}

Se trata de una guía dirigida al estudiante universitario que va a realizar prácticas académicas externas, en la que se recogen conceptos y pautas de actuación fundamentales en materia de Prevención de Riesgos Laborales. El objetivo último que persigue es evitar accidentes del alumnado durante el desarrollo de las prácticas y en caso de que se produzcan, el propio estudiante conozca el procedimiento de actuación que le permita mitigar los efectos adversos de la contingencia y acceder a una pronta asistencia sanitaria. Asimismo, este manual pretende ser una herramienta didáctica dirigida al profesorado universitario responsable de las prácticas académicas externas, para que la utilice como apoyo a la formación e información que debe transmitirse al estudiante previo al comienzo de sus prácticas.

En el manual, se abordan de forma transversal los contenidos relacionados con las prácticas de los estudiantes, entre otros: normativa de aplicación, tipos de prácticas, titulaciones y asignaturas afectadas, principales riesgos a los que se encuentran expuestos los estudiantes de Ciencias de la Salud, buenas prácticas y control de riesgos, procedimiento de actuación en caso de accidente, directorio y documentos de interés.

Asimismo, explica las herramientas que junto con el documento forman parte del proyecto de innovación docente: material audiovisual en el que se explican a través de vídeo los riesgos a los que se encuentran expuestos y las buenas prácticas preventivas dirigidas a estudiantes.

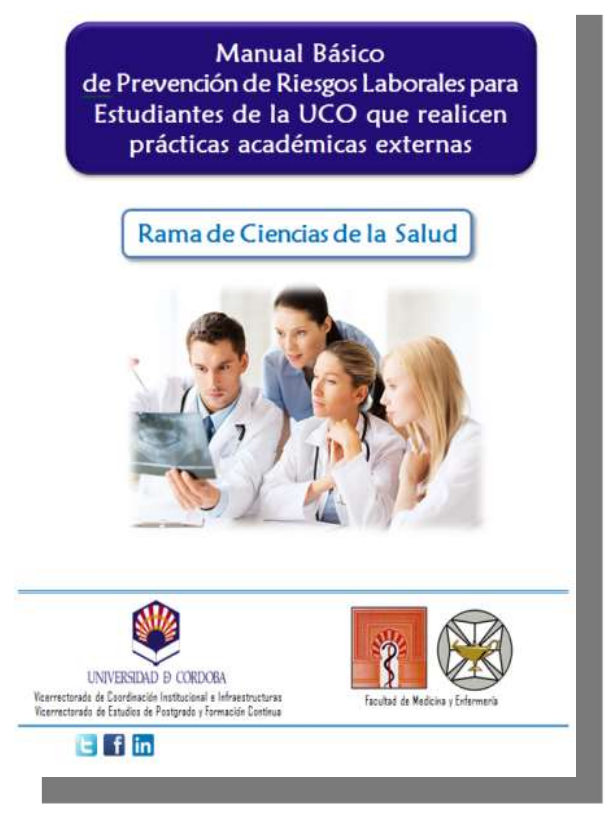

Imagen 1: Portada del Manual Básico de PRL.

Con relación a los riesgos, en el manual se han contemplado medidas preventivas sobre los riesgos mecánicos, químicos, físicos, biológicos, ergonómicos y psicosociales. 


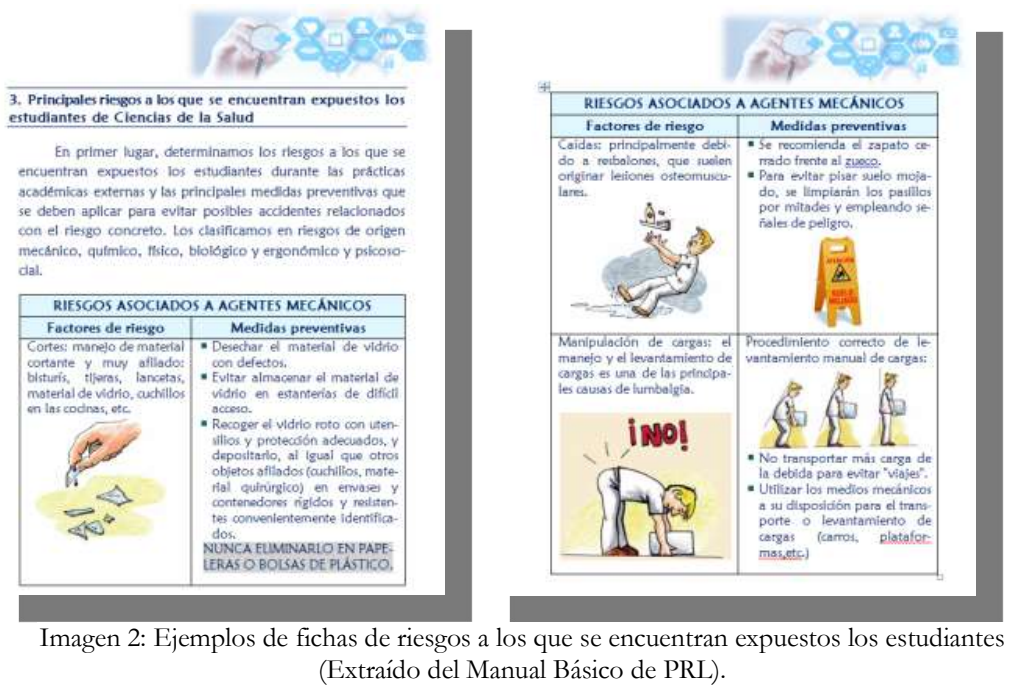

Por último, contiene una Declaración formativa e informativa en materia de prevención, que deberá ser firmada por el estudiante y el tutor académicos de la UCO, una vez que ha terminado su proceso formativo. En esta, se declara que el estudiante ha recibido formación e información teórica y práctica en materia de prevención de riesgos laborales aplicada a las prácticas académicas externas que va a realizar. En el mismo sentido, se establece que ha sido formado en buenas prácticas preventivas para evitar accidentes e informado sobre el procedimiento de actuación en caso de accidente durante el desarrollo de las prácticas.

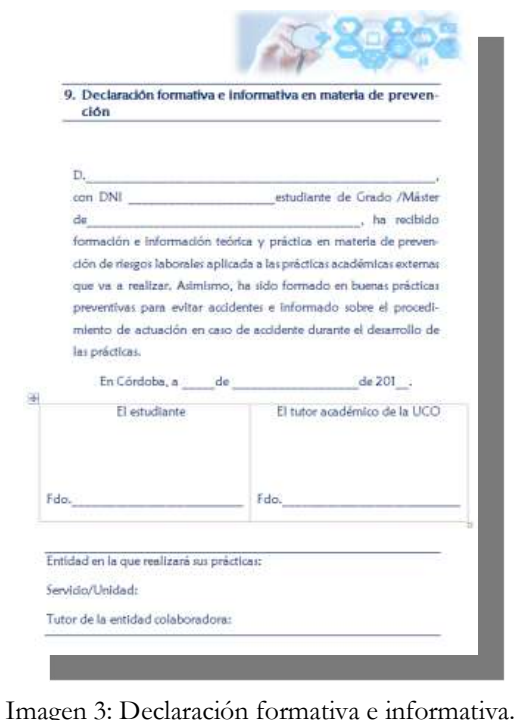

\subsection{MATERIAL AUDIOVISUAL Y APLICACIÓN INFORMÁTICA}

Se han elaborado contenidos audiovisuales y una herramienta informática que facilitan al estudiante la comprensión de los conceptos que deben conocer para que puedan desarrollar sus prácticas en un entorno seguro y saludable desde el punto de vista preventivo.

El material audiovisual consta de dos vídeos que reproducen parte de los contenidos del manual en forma de lección magistral, presentando mediante la exposición oral los aspectos clave de manera sintética, secuencial, motivadora y precisa.

- Vídeo 1: Principales riesgos a los que se encuentran expuestos los estudiantes de Ciencias de la Salud.

- Vídeo 2: Buenas prácticas y control de riesgos para estudiantes de Ciencias de la Salud.

La aplicación informática está basada en un entorno de gestión vía WEB y localizada, a través de un portal, en Internet.

Esta aplicación favorece que el estudiante vaya formándose autónomamente planteándose interrogantes, participando y asumiendo responsabilidades; y, por tanto, desarrollando la capacidad para aprender por sí mismo. Los contenidos del portal web son los siguientes: 1) ¿Qué son las prácticas académicas externas?; 2) Normativa de 
aplicación; 3) Vídeos: riesgos a los que están expuestos los estudiantes y buenas prácticas preventivas; 4) ¿Qué debo hacer en caso de accidente?; 5) Directorio; y 6) Documentos y enlaces de interés.

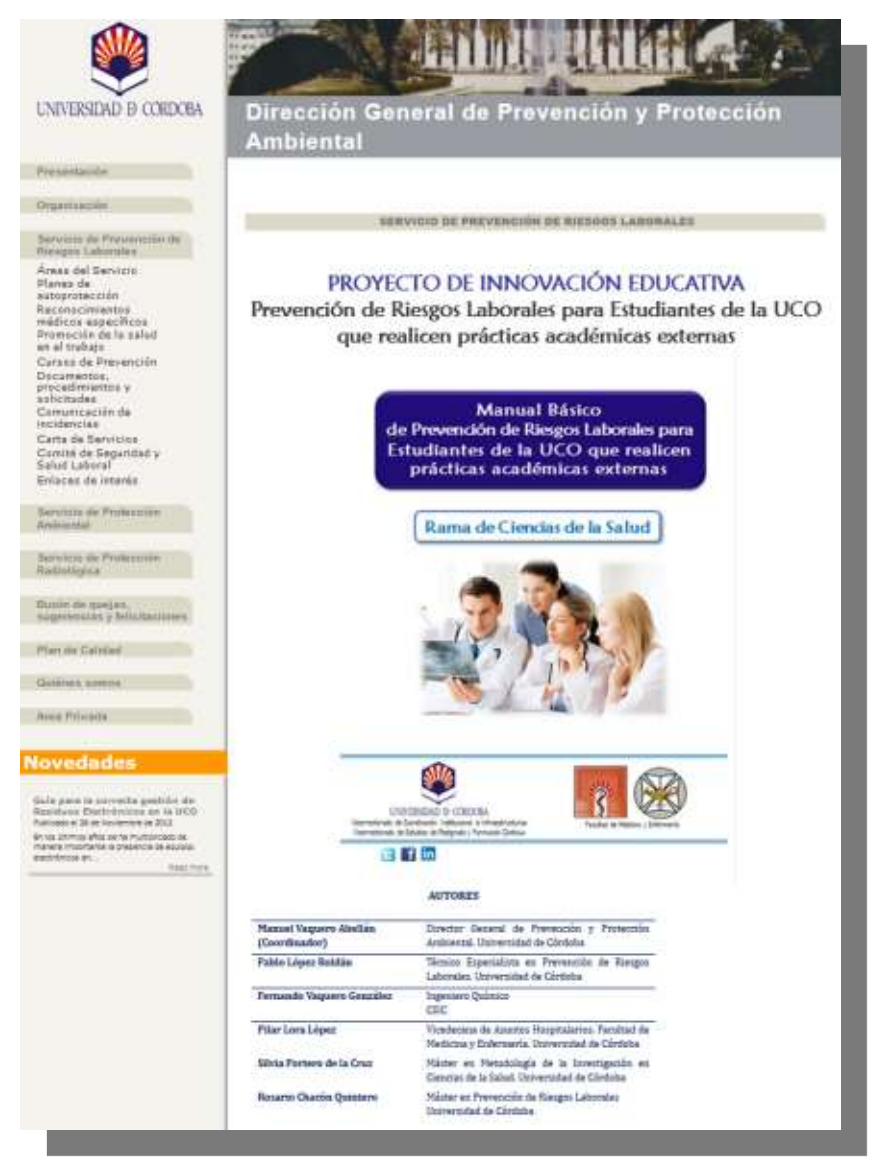

Imagen 4: Portal de acceso a la aplicación informática.

\section{UTILIDAD/ANÁLISIS}

Consideramos nuestra iniciativa fundamental para que el estudiante adquiera los conocimientos básicos en materia preventiva para desarrollar sus prácticas con las máximas garantías de seguridad y salud. Ha quedado demostrada la utilidad del mismo, puesto que es la principal vía a través de la que se pueden formar en esta materia, toda vez que las titulaciones carecen en muchos casos de asignaturas relacionadas con la prevención.

Con respecto a los contenidos audiovisuales y la plataforma informática, los estudiantes tienen acceso a una interacción virtual de gran utilidad y muy versátil, con contenidos específicos en materia de Prevención de Riesgos Laborales, que sin duda, facilita el aprendizaje de los conocimientos teóricos y prácticos en materia preventiva que se relacionan con las prácticas académicas externas.

\section{CONCLUSIONES/DISCUSIÓN}

Esta iniciativa favorece la formación autónoma y participativa de los estudiantes, consiguiendo que asuman responsabilidades y desarrollen la capacidad de aprender por sí mismos, de forma autocrítica. El manual complementario quiere ayudar al profesorado universitario responsable de las prácticas académicas externas, para que la utilice como herramienta de apoyo en la formación e información básica en prevención de riesgos que debe transmitirse al estudiante, previo al comienzo y durante la evolución de sus prácticas. 
BUENAS PRÁCTICAS PREVENTIVAS PARA ESTUDIANTES UNIVERSITARIOS DE CIENCIAS DE LA SALUD QUE REALICEN PRÁCTICAS ACADÉMICAS EXTERNAS

\section{BIBLIOGRAFÍA}

CAMPINS MARTÍ, M. “Enfermería, ¿profesión de riesgo?”, Medicina Clínica, 113, 1999, p. 699-700.

COMISIONES OBRERAS CASTILLA Y LÉON, SECRETARÍA DE SALUD LABORAL, Guía básica de riesgos laborales específicos en el Sector Sanitario. [Internet] [Consultado 15 octubre 2015]. Disponible en: http://www.sanidad.ccoo.es/comunes/recursos/15617/doc142620 Guia Basica de riesgos laborales en el sector sanitario.pdf

COMUNIDAD DE MADRID, Guía de acogida personal de Enfermería, [Internet]. Hospital Universitario Ramón y Cajal [Consultado 15 octubre 2015]. Disponible en:

http://www.madrid.org/cs/Satellite?blobcol=urldata\&blobheader=application $\% 2$ Fpdf\&blobheadername $1=$ Content-

disposition\&blobheadername $2=$ cadena\&blobheadervalue $1=$ filename $\% 3$ Dguia nuevaincorporacion 2006.pdf\&blobheadervalue $2=$ language $\% 3$ Des $\% 26$ site $\% 3$ DHospitalRamonCajal\&blobkey $=$ id \&blobtable =MungoBlobs\&blobwhere $=1202759386588 \&$ ssbinary $=$ true

COMUNIDAD VALENCIANA, Manual de acogida a alumnos de Enfermería Hospital General Universitario de Elda " $V$ irgen de la Salud". Centros de Atención Primaria. [Internet]. Agencia Valenciana de Salud. [Consultado 15 octubre 2015 ]. Disponible en:

https://fcsalud.ua.es/es/documentos/normativa/practicum/protocoloacogidaelda.pdf

COMUNIDAD VALENCIANA, Manual de acogida a estudiantes de Enfermería. [Internet]. Departamento de Salud de Elche. Hospital General. [Consultado 15 octubre 2015]. Disponible en:

https://fcsalud.ua.es/es/documentos/normativa/practicum/protocoloacogidaelche.pdf

ESPAÑA. Ley 31/1995, de 8 de noviembre, de Prevención de Riesgos Laborales. Boletín Oficial del Estado, 10 de noviembre de 1995, núm. 269, p. 32590.

ESPAÑA. Ley de 17 de julio de 1953 (Jefatura del Estado), sobre el establecimiento del Seguro Escolar en España. Boletin Oficial del Estado, 18 de julio de 1953, núm. 199.

ESPAÑA. Real Decreto 1791/2010, de 30 de diciembre, por el que se aprueba el Estatuto del Estudiante Universitario. Boletín Oficial del Estado, 31 de diciembre de 2010, núm. 318, p. 109353.

ESPAÑA. Real Decreto 592/2014, de 11 de julio, por el que se regulan las prácticas académicas externas de los estudiantes universitarios. Boletín Oficial del Estado, 30 de julio de 2014, núm. 184, p. 60502.

FREMAP MUTUA COLABORADORA CON LA SEGURIDAD SOCIAL No 61, Manual de Seguridad y Salud en el Sector Hospitales. [Internet] [Consultado 15 octubre 2015]. Disponible en: http://prevencion.fremap.es/Buenas $\% 20$ prcticas/MAN.013\%20(castellano) $\% 20-020$ M.S.S. $\% 20$ Sector $\% 20 H_{0}$ spitalario.pdf

FREMAP MUTUA COLABORADORA CON LA SEGURIDAD SOCIAL No 61, Manual de Seguridad y Salud frente al Riesgo de Exposición Laboral a los Campos Electromagnéticos en los puestos de Fisioterapeuta y Auxiliar. [Internet] [Consultado 15 octubre 2015]. Disponible en: http://prevencion.fremap.es/Buenas $\% 20$ prcticas/LIB.014\%20\%20M.S.S. $\% 20$ Riesgos $\% 20$ Campos $\% 20$ Electromagneticos.pdf $^{2}$

JUNTA DE ANDALUCÍA. Acuerdo de 3 de octubre de 1995, del Consejo de Gobierno, por el que se autoriza la suscripción de un Convenio-Marco entre las Consejerías de Salud y Educación y Ciencia y las Universidades de Almería, Cádiz, Córdoba, Granada, Huelva, Jaén, Málaga, Sevilla, para la utilización de las Instituciones Sanitarias en la investigación y la docencia. Boletín Oficial de la Junta de Andalucía, 21 de noviembre de 1995, núm. 148, p. 10908.

JUNTA DE ANDALUCÍA. Resolución de 31 de julio de 2000, de la Universidad de Córdoba, por la que se da publicidad al Concierto entre la Junta de Andalucía y la Universidad de Córdoba para la utilización de las Instituciones Sanitarias en la Investigación y la Docencia. Boletín Oficial de la Junta de Andalucía, 5 de septiembre de 2000, núm. 102, p. 14196.

REGIÓN DE MURCIA, Manual de Acogida y Funcionamiento del Servicio de Quirófano para Profesionales de Nueva Incorporación. [Internet]. Consejería de Sanidad. Servicio Murciano de Salud. [Consultado 15 octubre 2015]. Disponible en: https://www.murciasalud.es/recursos/ficheros/260549-manual quirofano1.pdf

UNIVERSIDAD DE CÁDIZ, DIRECCIÓN GENERAL DE CIENCIAS DE LA SALUD, Documento de acogida para la realización de prácticas clínicas de los estudiantes de ciencias de la salud, [Internet] Facultad de Medicina. [Consultado 
15 2015]. Disponible $\quad$ http://medicina.uca.es/wdocencia/practicas-

clinicas $/$ Documento $\% 20$ de $\% 20$ acogida $\% 20$ para $\% 2012 \% 20$ realizaciyn $\% 20$ de $\% 20$ practicas $\% 20$ clinicas.pdf

UNIVERSIDAD DE CÓRDOBA, Convenio especifico de colaboración entre la Universidad de Córdoba y el Hospital Universitario Reina Sofía, para la realización de prácticas de alumnos tuteladas, Córdoba, 2001.

UNIVERSIDAD DE CÓRDOBA, Acuerdo de Consejo de Gobierno, en su sesión ordinaria de 20/07/2012, por el que se aprueba la Normativa para el desarrollo de las prácticas clínicas del título de Grado en Enfermería, Boletín Oficial de la Universidad de Córdoba, 29 de octubre de 2014, núm. 102.

UNIVERSIDAD DE CÓRDOBA, Procedimiento de actuación en caso de Accidentes en el Desarrollo de las Prácticas Clinicas. [Internet]. Facultad de Medicina y Enfermería. [Consultado 15 octubre 2015]. Disponible en: http:/ /www.uco.es/medicina/sites/default/files/archivos/documentos/enfermeria/practicasexternas/Procedimiento Actuacion Accidentes en Desarrollo Practicas Clinicas-triptico.pdf

UNIVERSIDAD DE LAS PALMAS DE GRAN CANARIA, Manual de Acogida al Estudiante en Materia de Prevención de Riesgos. [Internet]. Servicio de Prevención de Riesgos Laborales. [Consultado 15 octubre 2015]. Disponible en: http://www.sprl.ulpgc.es/index.php/30-sprlaborales/noticias/difusion-informacion/54-manual-de-acogida-al-estudiante-en-materia-de-prevencion-de-riesgos

UNIVERSIDAD DE MURCIA, Manual de prácticas tuteladas. [Internet]. Departamento de Fisioterapia. [Consultado 15 octubre 2015]. Disponible en:http://www.um.es/documents/115466/2963017/Manual+de+Pr\% $\%$ C3\% Alcticas + Tuteladas Noviembre2015.pdf/808873ecdb47-49e3-9d44-afb63cf33f5a

UNIVERSIDAD POLITÉCNICA DE VALENCIA, Guia de planificación de prácticas de alumnos en laboratorios con riesgos quimicos. [Internet]. [Consultado 15 octubre 2015]. Disponible en: https://www.sprl.upv.es/pdf/gu $\%$ EDa $\% 20$ pr $\%$ E1cticas $\% 20$ alumnos $\% 20$ riesgo\% $\% 2$ qu $\%$ EDmico.pdf 